\title{
THE EFFECTS OF THE PARAMETERS OF 3D PRINTING IN AN ELECTRICAL MACHINE
}

\author{
Dénes Tóth \\ PhD Student, University of Miskolc, Institute of Materials Science and Engineering, \\ Department of Mechanical Technologies \\ 3515 Miskolc, Miskolc-Egyetemváros, e-mail: toth.denes@uni-miskolc.hu \\ Miklós Tisza \\ Professor emeritus, University of Miskolc, Institute of Materials Science and Engineering, \\ Department of Mechanical Technologies \\ 3515 Miskolc, Miskolc-Egyetemváros, e-mail: tisza.miklos@uni-miskolc.hu
}

\begin{abstract}
In recent year, the e-mobility is undergoing an exponential grow because of $\mathrm{CO}_{2}$ restrictions. Until 2025 it is expected to be between 7-14\% of the total market share, which means that combustion engines have to be more efficient to be competitive. Start-stop and $48 \mathrm{~V}$ hybridization are rapidly increase market share, to help reaching $\mathrm{CO}_{2}$ legislation targets. Every possibilities has to be investigated to increase the efficiency: in this paper, the consequences of using $3 D$ metal printed parts in electrical machine will be examined from electrical aspect. [1]
\end{abstract}

Keywords: 3D printing, electrical machine, alternator, magnetization, claw-pole

\section{Introduction}

Development pressures on automotive manufacturers and automotive suppliers are constantly increasing: environmental demands and user demands are rising at the same time. The main objective of the development of electric and hybrid drives, as well as to spur car manufacturers to develop cleaner, more efficient internal combustion engines. The e-mobility in contrast to previous pre-signals more slowly gaining ground in the market, so further improvements to internal combustion engines are even more important in the near future. The development of the internal combustion engine auxiliary equipment designed to reduce total energy use of vehicles: the start-stop and hybrid systems $48 \mathrm{~V}$ or greater efficiency and generators that serve these purpose [1].

\section{The alternator}

The generator is an energy conversion device of all combustion engines that absorbs a portion of the vehicle's mechanical energy and converts it into electrical current for on-board consumers. The pulley on the rotor shaft establishes a connection between the crankshaft of the engine and the rotor by means of a belt drive. The ratio of mechanical input and electrical output is regarded as the efficiency.

The rotor is an electromagnet: the winding inside is excited by a voltage regulator, which magnetizes the pair of claw poles through the iron core of the coils, creating a rotating magnetic field. This rotating magnetic field generates a sinusoidal voltage in its sinusoidal distributed wires along the stator 
circumference. The disadvantage is that the resulting current is AC, so rectification is needed to supply the vehicle's electrical system and battery power.

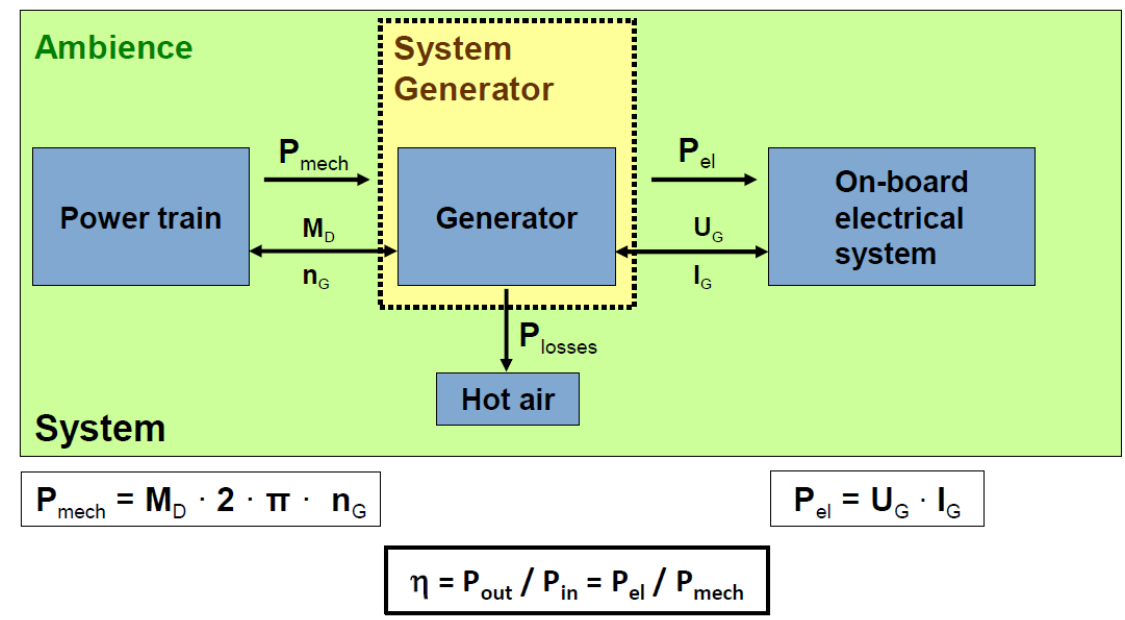

Figure 1. The schematic figure of an alternator how the alternator converts the mechanical to electrical energy [2]

The subject of our study is the claw pole, which is an important component of the electrical machine. As demonstrated by Upadhayay [5] et al. in their simulation studies, they were able to greatly influence the local magnetic flux density within the material by changing the main geometric parameters. The more uniform the distribution of flux within the component, the more ideal it is for the efficiency of the machine. The raw material of the part is C4C (EN 1.0303) grade unalloyed steel due to its good magnetic properties.

Table 1: Material composition of C4C (1.0303) steel [\%], [3]

\begin{tabular}{|c|c|c|c|c|c|c|}
\hline Material & C & Si & Mn & P & S & Al \\
\hline C4C (1.0303) & 0.05 & 0.04 & 0.38 & 0.02 & 0.025 & 0.044 \\
\hline
\end{tabular}

\section{The new manufacturing process}

The currently used claw poles are produced by cold and hot forging: it is intended to be replaced with 3D printed pieces of the same material, as a result of our research.

To accomplish this, we use the ORLAS Creator 3D Printer of the Institute of Casting, Faculty of Materials Engineering, at the University of Miskolc. The machine operates on the principle of laser sintering: it places on the work table a metal layer of a given thickness, which is melted according to the desired geometry in the relevant area of the layer with laser light in the presence of argon/nitrogen and then repeated throughout the geometry. 


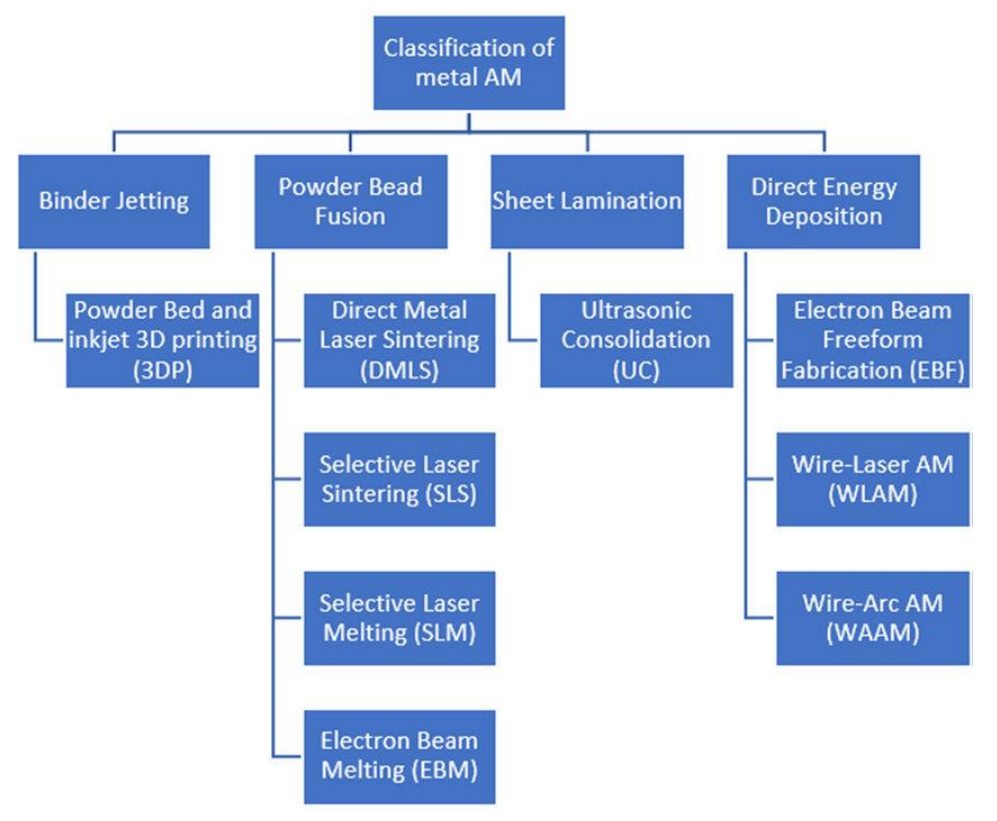

Figure 2. The classification of metal additive manufacturing processes [10]

The following print settings are treated as independent variables in our experiment:

- Print orientation

- Particle size

- Print layer thickness

- Raw material (possibly additional alloying)

- The existence and method of post heat treatment of the parts.

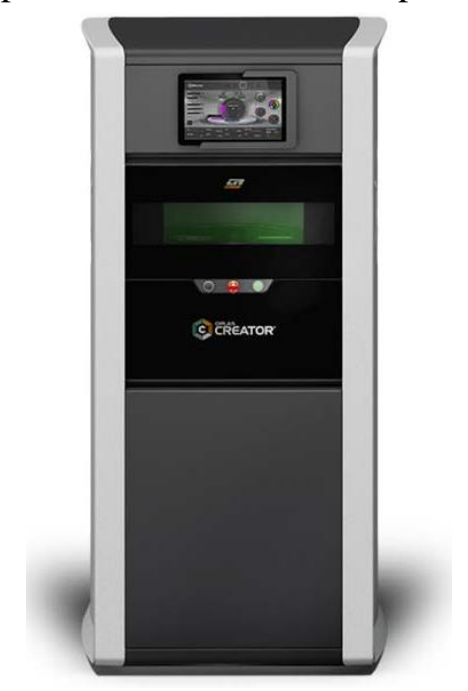

Figure 3. The ORLAS Creator metal 3D printer [7] 
The huge advantage of the this equipment over most of others that are currently available on the market is that it can process not only alloys recommended by the manufacturer, but various types of raw materials (metals), including the current $\mathrm{C} 4 \mathrm{C}$ grade steel, too.

Another equipment, the ATO Lab Atomizer will be used (located at the Institute of Foundry) to produce the raw material for the printing. The machine applies ultrasonic atomization technology: first to create a plasma sheet by tungsten electrode, in which the fed metal wire is melted, and then, at a given frequency, sonicated by excitation, then drops into particles and solidifies in the form of metal powder. The input parameters influence the size of the resulting solid particles, which can be further separated and classified by sieving.

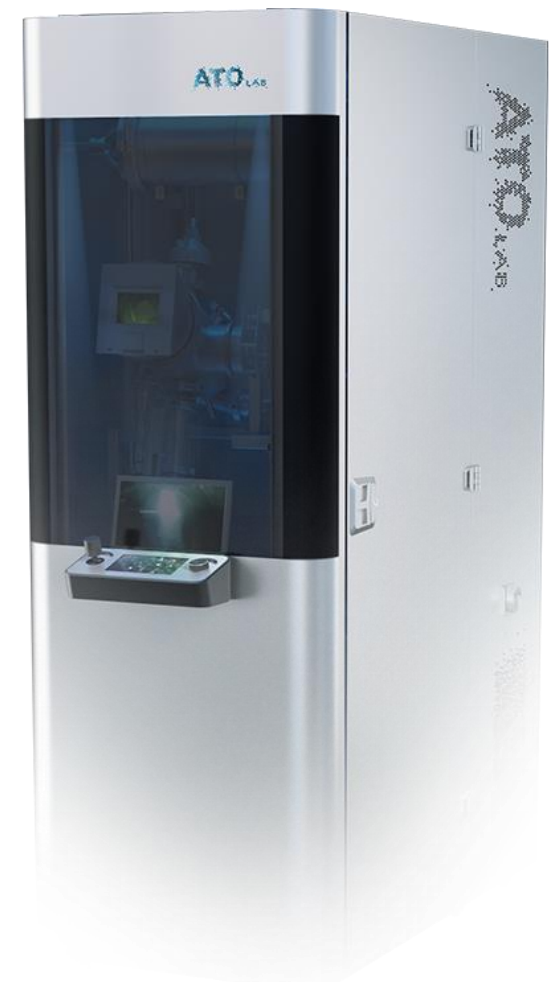

Figure 4. The ATO Lab atomizer machine [6]

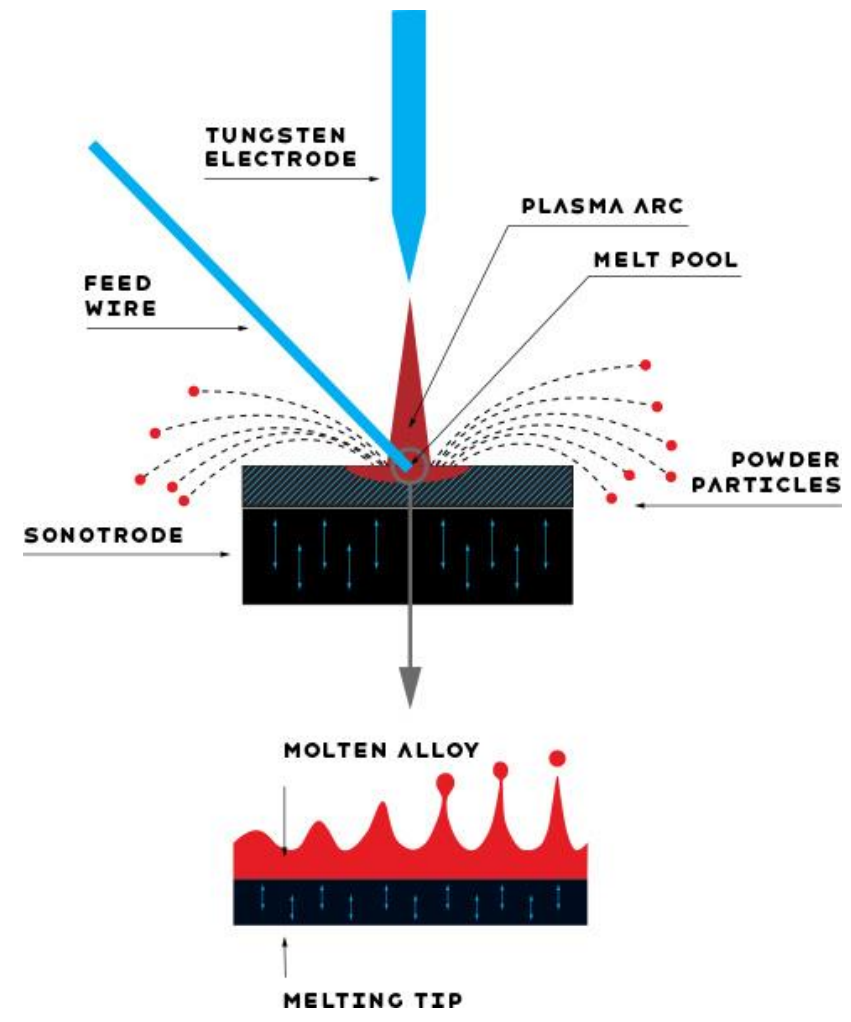

Figure 5. A sketch of the working principle of ATO Lab atomizer [6]

\section{Planned investigations - Robograph and finite element analysis}

When a ferromagnetic material is placed in a magnetic field, its particles will be aligned with the orientation of the magnetic field; the extent of this alignment can be measured. 


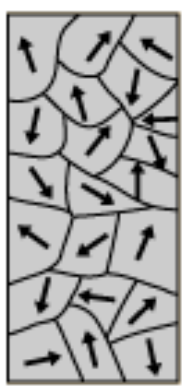

In bulk material the domains usually cancel, leaving the material unmagnetized.
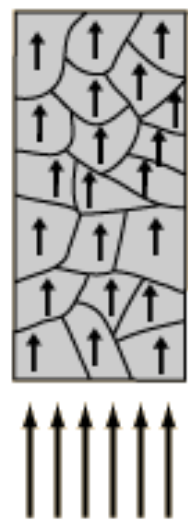

Externally applied magnetic field.
Iron will become magnetized in the direction of any applied magnetic field. This magnetization will produce a magnetic pole in the iron opposite to that pole which is nearest to it, so the iron will be attracted to either pole of a magnet.

Figure 6. The domains arrangement according to the magnetic field orientation [8]

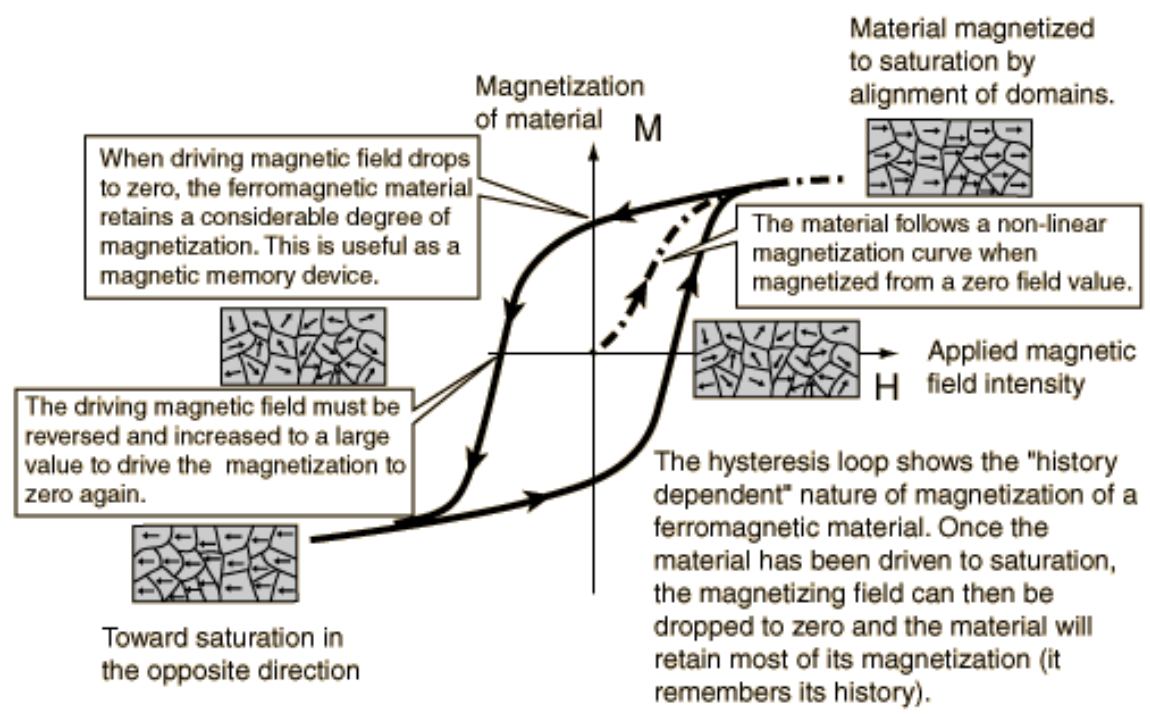

Figure 7. The change in the arrangement of domains in during the hysteresis measurement [8]

During the test, the specimen is subjected to a magnetic field ( $\mathrm{H}$, magnetic field strength) so that the initially magnetically neutral piece is magnetized. The magnetization of the material $(\mathrm{M})$ reaches a saturation point, from which the magnetization of the material no longer increases significantly. The saturation point is often referred to as an elbow point, that is a prominent point on the hysteresis curve, so the field strength at this point is also noted separately, called boundary field strength. Subsequently, by reversing the excitation poles, the material is first magnetized, and the associated opposite field strength is called coercive field strength. Subsequently, the material is further ordered to the opposite 
point of saturation, and after another pole reversal, we return to the point of first magnetization: the path thus described regarded as the hysteresis curve of the specimen. This shows the susceptibility of the substance as follows:

$$
M=\chi * H,
$$

where:

$$
\begin{array}{ll}
M & \text { magnetization of the material }\left[\frac{A}{m}\right], \\
H & \text { magnetic field strength }\left[\frac{A}{m}\right] .
\end{array}
$$

This shows the extent to which the material is magnetized by the exposed magnetic field. The specimens produced in different ways result in different magnetic susceptibilities, thus affecting the propagation of the magnetic flux within the material, and ultimately the electrical power and efficiency of the machine.

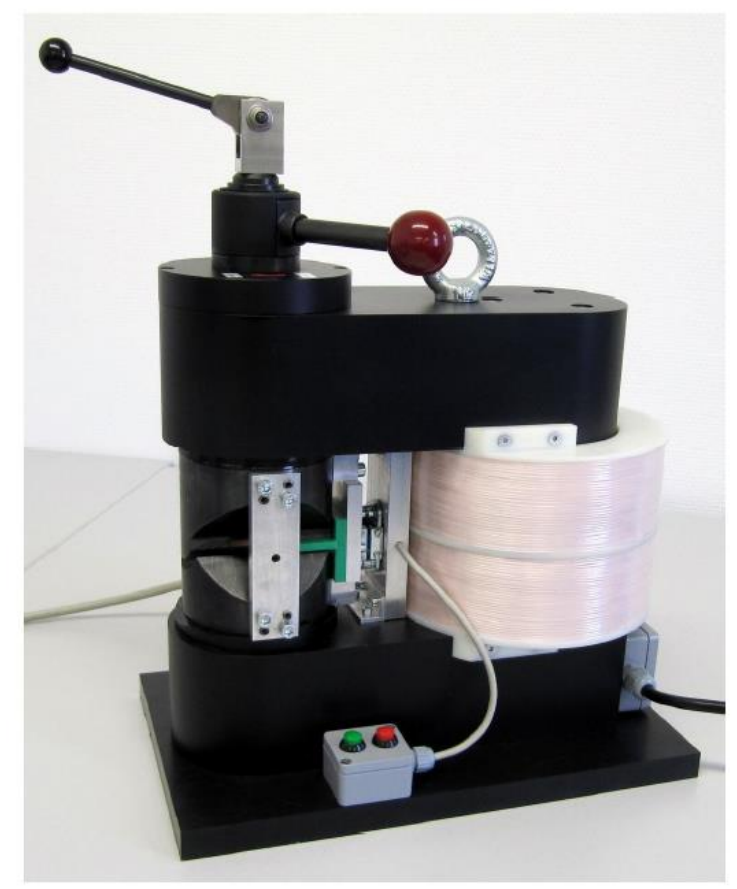

Figure 9. ECKEL Robograph RE flux hysteresis measuring device

The Robograph equipment from SEG Automotive Ltd.'s fleet will be used to measure these properties. The machine is capable of taking the hysteresis curve of the manufactured specimens and the orthotropic magnetic properties of the material as described. The magnetic properties determined in this way can now be used in the Ansys Maxwell finite element environment, so we can model not only the effects of geometric parameters, but also of SLS manufacturing technology. The advantage of this is that there is no need to physically produce all of the pieces, the electrically ideal parts can get earlier into focus, it is enough to actually produce the promising ones. 
Also, through the facilities provided by the SEG Automotive Ltd, we are able to verify the simulation results of the performance tests, temperature tests and other physical efficiency tests that can be carried out on a series of test equipment available for physical validation of our study.

\section{Summary}

The subject of this research is the changing of the manufacturing technology of a claw pole on a rotor of an alternator, used in passenger cars. The current forged claw poles made of $\mathrm{C} 4 \mathrm{C}$ steel may be compared to those ones that are made with a $3 \mathrm{D}$ printer using the same material, primarily in terms of electrical properties.

To do this, we first need to make specimens with the 3D printer with different input parameters to investigate their effects on magnetization. The resulting material data can be used in a finite element environment, where we can investigate the effect of the new manufacturing process on electrical machine performance, which will be certified by the available physical tests.

\section{Literature}

[1] SEG Automotive Whitepaper - https://www.seg-automotive.com/de/news//asset_publisher/7e2eIV4ftufX/content/whitepaper-transformation-of-the-powertrain

[2] SEG Automotive Training material 2019

[3] Material datasheet- Saarstahl - https://www.saarstahl.com/sag/downloads/download/15209

[4] Coercivity and Remanence in Permanent Magnets, http://hyperphysics.phyastr.gsu.edu/hbase/Solids/magperm.html

[5] Pranshu Upadhayay, Afef Kedous-Lebouc , Lauric Garbuio.: Impact of Claw-Pole Geometry Variations on the Performance of Machine used in Automotive, Application Univ. Grenoble Alpes, CNRS, Grenoble INP, G2Elab, F-38000 Grenoble, France

[6] http://metalatomizer.com/\#features

[7] https://creator.or-laser.com/en/

[8] https://www.imakr.com/uk/or-laser/orlas-creator-metal-3d-printer

[9] http://hydrogen.physik.uni-wuppertal.de/hyperphysics/ hyperphysics/hbase/solids/ hyst.html

[10] Li, J.L.Z., Alkahari, M.R., Rosli, N.A.B., Hasan, R., Sudin, M.N., Ramli, F.R. : Review of Wire Arc Additive Manufacturing for 3D Metal Print International Journal of Automation Technology 2019, 13(3):346-353. https://doi.org/10.20965/ijat.2019.p0346 